\title{
Ultrasonic diagnosis of diffuse liver disease
}

The hepatitis C virus (HCV) was discovered in 1989, and in the same year it became possible to measure levels of $\mathrm{HCV}$ antibodies at leading medical facilities. In the following year, a technique for the definite diagnosis of hepatitis $\mathrm{C}$ became available across Japan. This was an epoch-making event for clinicians involved in the management of liver disease. The discovery of HCV triggered rapid advances in research on the detailed pathophysiology of "non-A, non-B hepatitis," which had been difficult to study before; it was as if investigators had found the key to unlocking the problem. At the same time, remarkable progress was made toward treatment of this type of hepatitis. Following the revolutionary discovery of this disease, which accounts for the overwhelming majority of all cases of liver disease in Japan, approaches to clinical management of liver disease also underwent considerable changes.

During the "non-A, non-B" period, clinicians were occupied with the idea that estimation of the current status and prediction of the prognosis of this type of liver disease were nearly impossible because of its unknown etiology. Despite this underlying idea, clinicians in those days did try every means available at the time to assess the current status of the disease and its changes over time. The means employed varied from physician to physician. My approach was to utilize a combination of ultrasonography and liver biopsy. The conditions called "non-A, non-B liver disease" included not only a large group of post-transfusion hepatitis but also autoimmune hepatitis, drug-induced hepatitis, alcoholic liver injury, fatty liver, and primary biliary cirrhosis. Thus, the term "non-A, non-B liver disease" encompassed all of those liver diseases that were difficult to diagnose unless the physician was active in or passionate about this particular field. For this reason, ultrasonic tissue characterization was useful when dealing with this disease. Noninvasive ultrasonography is quite useful as a means of assessing changes over time. Of course, liver biopsy is performed in the early stages of the disease to obtain information that will serve as a baseline for subsequent follow-up. In subsequent stages, ultrasonography is carried out at regular intervals to check for changes in liver parenchyma, the entire liver morphology, and surrounding organs. As needed, liver biopsy is performed again. The physician taking these steps can progressively correlate the ultrasonic findings with the histological features, and thus the need for invasive liver biopsy is gradually reduced. This is a major advantage of ultrasonic tissue characterization. In those days, a number of reports pertaining to this topic were published, and remarkable advances were made in ultrasonic tissue characterization. The knowledge and findings collected in that period have been passed down without interruption and have been utilized clinically. "Is the hepatopathy in this case attributable to fat, alcohol, congestion, or biliary obstruction?" "Has chronic hepatitis in this case advanced to the stage of cirrhosis?" When we face such questions, ultrasonography can immediately provide valuable information. The fact that ultrasonography allows us to look inside the body quite easily is attractive to clinicians.

As the methods of treatment for hepatitis $\mathrm{C}$ advanced after the discovery of HCV, however, the necessity of conducting detailed tissue characterization in an enthusiastic manner gradually decreased among clinicians dealing with cases of hepatitis C. HCV-positive cases of hepatitis are indicated for interferon therapy. If treatment succeeds and $\mathrm{HCV}$ disappears, everything is fine. If $\mathrm{HCV}$ eradication cannot be achieved, treatment needs to be repeated so long as it is financially and physically feasible. If repeated treatment does not succeed, then the target is set at dealing with cirrhosis or liver cancer. I sense that during such a course of management, physicians are no longer likely to feel the necessity of detailed tissue characterization. One favorable trend is that the level of liver fibrosis has recently begun to be taken into account when hepatitis $\mathrm{C}$ is managed. It is gratifying that research on ultrasonic assessment of fibrosis of liver parenchyma has been stimulated by this trend.

Although we have been through a period when substantial progress on the development of ultrasonic tissue characterization seemed to have stalled, encouraging news in this field has recently been reported. For example, it was shown that ultrasonography with an intravenous ultrasound contrast agent (an agent first marketed about 10 years ago) can yield images with quite high spatial resolution not pos- 
sible with any other modality of diagnostic imaging. The contrast agent Sonazoid, most frequently used at present, can be used only for hepatic tumors under the national health insurance system in Japan, and its use for diagnosis of diffuse liver disease is not yet covered by insurance. However, clinical trials on this agent in a small number of patients, conducted under authorization of the institutional review board, are yielding very promising results related to small vessels within the liver parenchyma and microcirculation. ${ }^{1,2}$ Furthermore, although not yet widely known, remarkable advances in the devices used for ultrasonography have been stimulated by development of such ultrasound contrast agents. The advances achieved to date include improvement in B-mode imaging (thanks to techniques such as harmonic imaging), elevation in resolution, and improvement in Doppler sensitivity, which have made it possible to obtain diverse and useful information by ultrasonography even when no contrast agent is used. Furthermore, in connection to the above-mentioned attempt at fibrosis assessment, new techniques have been developed that allow immediate visualization or numerical representation of tissue distortion caused by application of a certain force, and these techniques are now close to the stage of full-scale clinical application. ${ }^{3}$ In addition, attempts have been made to extract subtle or detailed information about tissues from the speckle signals filling the background of B-mode images. ${ }^{4,5}$
At present, routine clinical management of various liver diseases (including hepatitis C) involves the steps of diagnosis, observation of the natural course, observation after therapeutic intervention, and observation of liver function and blood flow and monitoring of portal hypertension and other parameters at advanced stages of the disease. There seem to be many occasions during the management of liver disease at which clinicians should try to actively collect information. Ultrasonography has an unlimited potential to be utilized on such occasions. We can expect much of ultrasonography following further maturation of the devices, contrast agents, and techniques related to ultrasound.

\section{References}

1. Yoshizawa K, Fujita Y, Nakajima S, et al. Evaluation of the liver blood flow by using Sonazoid-enhanced ultrasound. Rad Fan 2007;5(7):47-9 (in Japanese).

2. Sumino Y. Ultrasound update: evolution of flow imaging, part 1. Contrast-enhanced imaging - liver diseases. Clin Imagiol 2008; 24(5):558-63 (in Japanese).

3. Saito M. Virtual palpation. J Med Ultrasound Technol 2008;33(6): 659-65 (in Japanese).

4. Kamiyama N, Yamaguchi T, Hachiya H. Tissue characterization using statistical information from ultrasound echo signals. Med Imaging Technol 2003;21(2):112-6 (in Japanese).

5. Sumino Y, Fujita Y, Yoshizawa K, et al. Ultrasound imaging of liver diseases. Kan Tan Sui 2007;55(4):619-32 (in Japanese). 